\title{
Lasing Modes of Infinite Periodic Chain of Quantum Wires
}

\author{
Volodymyr O. Byelobrov ${ }^{1}$, Trevor M. Benson ${ }^{2}$, Phillip Sewell ${ }^{2}$, Ayhan Altintas ${ }^{3}$, and Alexander I. Nosich ${ }^{1}$ \\ ${ }^{1}$ Institute of Radiophysics and Electronics NASU, ul. Proskury 12, Kharkiv 61085, Ukraine \\ Tel:+380(57)7203782 e-mail: volodia.byelobrov@gmail.com \\ ${ }^{2}$ George Green Institute for Electromagnetics Research, University of Nottingham, Nottingham, NG7 2RD, UK \\ ${ }^{3}$ Bilkent University, Ankara 06800, Turkey
}

\begin{abstract}
In this paper, we study the scattering and eigenvalue problems for a periodic open optical resonator that is an infinite chain of active circular cylindrical quantum wires standing in free space. The scattering problem is solved by the method of partial separation of variables. The eigenvalue problem differs from the first one by the absence of the incident field and presence of "active properties" of cylinders and yields the frequencies and thresholds of lasing.
\end{abstract}

Keywords: scattering. eigenproblem, laser, microcavity, quantum wire, threshold, Floquet series.

\section{INTRODUCTION}

Today's technologies enable manufacturing of advanced light-emitting devices based on single or multiple quantum wires embedded in epitaxially grown semiconductor microcavities [1]. Advantages of quantum wires with respect to quantum wells include their better thermal stability, lower chemical reactivity, and higher mechanical strength..

\section{PROBLEM FORMULATION}

The considered resonator consists of the parallel to the $z$-axis and periodic along the $x$-axis circular cylinders see Fig. 1. The distance between cylinder centers is $p$ and their radii are $a$. We consider that the electromagnetic field is time-harmonic $\left(\sim e^{-i \omega t}\right)$ and does not vary along the $z$-axis.

Then two alternative polarizations, $\mathrm{E}$ and $\mathrm{H}$, can be considered separately using the function, $U(x, y)$, which will denote the $E_{z}$ or $Z_{0} H_{z}$ component of the electromagnetic field, respectively. It should satisfy the Helmholtz equation with different coefficients: $\left(k^{\prime}\right)^{2}=k^{2} v^{2}$, where $\quad v=\alpha$ in scattering problem and $v=\alpha-i \gamma$ in eigenproblem $(\alpha, \gamma>0)$, inside each cylinder and $k^{2}$ outside. Here, $\alpha$ is the refractive index and $\gamma$ is the material gain which appears in the presence of pumping - see [2] for detailed

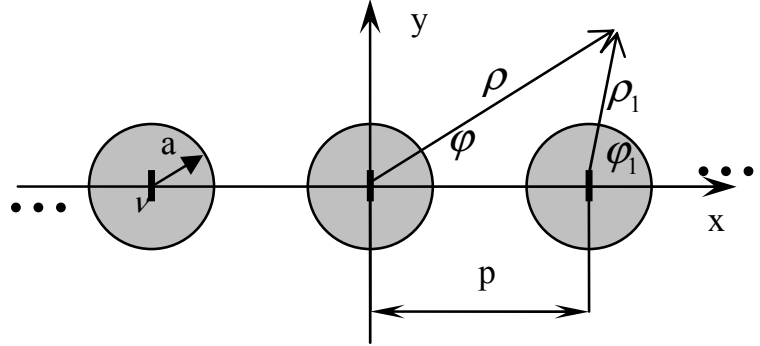

Fig. 1. Sketch of the periodic cavity of active dielectric circular cylinders or quantum wires. discussion of the linear eigenproblem approach in the linear modelling of microcavity lasers. Note also that it had been applied in the earlier papers $[3,4]$ to the threshold analysis in the 2-D circular microcavities and in [5] to the similar analysis of the 1-D VCSEL-type layered cavity containing an active region.

The field components tangential to cylinder's boundary must be continuous. Besides, $U$ must satisfy the condition of the local power finiteness and the condition of radiation at infinity. Although the most general situation implies that the field functions within the adjacent elementary cells of the periodic cavity differ by a phase factor, i.e. $U(x+p, y)=U(x, y) e^{i \beta p}$, in the following treatment we will assume that $\beta=0$. The task is to find non-zero solutions to the formulated homogeneous problem.

\section{BASIC EQUATIONS FOR SCATTERING PROBLEM}

\subsection{Boundary conditions}

The periodicity condition means that internal fields of cylinders are equal to each other, and the contributions to the external field generated by each of cylinders are the same. Hence, on introducing the local polar coordinates co-axially with each cylinder as $\left(r_{s}, \varphi_{s}\right)$ and the global coordinates with the origin in the centre of the 0 -th cylinder as $(r, \varphi)$, we can use the partial separation of variables and write

$$
U_{s}^{\text {int }}\left(r_{s}, \varphi_{s}\right)=\sum_{(n)} c_{n}^{\prime} J_{n}\left(k^{\prime} r_{s}\right) e^{i n \varphi_{s}}, \quad r_{s}<a
$$




$$
U^{\text {out }}(r, \varphi)=\sum_{(s)} U_{s}^{\text {out }}\left(r_{s}, \varphi_{s}\right)=\sum_{(s)} \sum_{(n)} b_{n}^{\prime} H_{n}^{(1)}\left(k r_{s}\right) e^{\text {in } \varphi_{s}}, \quad r_{s}>a
$$

where summations are performed from $-\infty$ to $+\infty$. Note that expressions (1) and (2) satisfy the Helmholtz equation and the conditions of radiation and local power finiteness.

The incident field that propagates alongside axis-y and comes from the positive direction may be written in following

$$
U^{i n c}=\sum_{(n)}(-1)^{n} J_{n}(k r) e^{i n \varphi} .
$$

The series (2) for the external field is not convenient because of the presence of arguments expressed in different origins. To bring formulation to the global-coordinate origin we apply the addition theorem for cylindrical functions,

$$
H_{n}^{(1)}\left(k \rho_{s}\right) e^{i n \varphi_{s}}=\sum_{(l)} H_{n-l}^{(1)}(k s p) J_{l}(k \rho) e^{i n \varphi} .
$$

This leads to the representation that contains infinite sums of the Hankel functions:

$$
U^{\text {out }}(\rho, \varphi)=\sum_{(n)} b_{n} H_{n}^{(1)}(k \rho) e^{i n \varphi}+\sum_{(n)} b_{n} \sum_{s^{\prime} \neq 0} \sum_{(l)} H_{n-l}^{(1)}(k s p) J_{l}(k \rho) e^{i l \varphi} .
$$

Continuity conditions on cylinder's boundary bring us to two coupled infinite matrix equations with the set of unknowns $\left\{c_{m}\right\}_{m=-\infty}^{\infty}$ and $\left\{b_{m}\right\}_{m=-\infty}^{\infty}$,

$$
\begin{aligned}
& c_{m} J_{m}\left(k^{\prime} r\right)=b_{m} H_{m}^{(1)}(k r)+\sum_{(n)} b_{n} J_{m}(k r) L_{m-n}+(-1)^{m} J_{m}(k r), \\
& \left(\frac{k^{\prime}}{k}\right)^{ \pm 1} c_{m} J_{m}^{\prime}\left(k^{\prime} r\right)=b_{m} H_{m}^{(1)^{\prime}}(k r)+\sum_{(n)} b_{n} J_{m}{ }^{\prime}(k r) L_{m-n}+(-1)^{m} J_{m}^{\prime}(k r),
\end{aligned}
$$

where the sign "+" corresponds to E-polarization and the "--" does to H polarization, $L_{m}$ is so-called lattice sums, those in the case of normal incidence vanish for odd order and are

\subsection{Lattice Sums}

$$
L_{m}(k p)= \begin{cases}2 \sum_{s>0} H_{m}(s k p), & m=2 k \\ 0, & m=2 k+1\end{cases}
$$

The lattice sums are treated by Twersky's approach that is a summation using Bernoulli coefficients

$$
\begin{aligned}
& L_{0}(x)=-1-\frac{2 i}{\pi}\left(\gamma+\log \frac{x}{4 \pi}\right)+\frac{2}{x}+2 \sum_{m>0}\left(\frac{2}{\sqrt{x^{2}-(2 \pi m)^{2}}}+\frac{i}{m \pi}\right) \\
& L_{2 l}(x)=4 \sum_{m=0}^{\infty} \frac{e^{-2 i l \arcsin \left(\frac{2 m \pi}{x}\right)}}{\sqrt{x^{2}-(2 \pi m)^{2}}}+\frac{2}{x}+\frac{i}{l \pi}+\frac{i}{\pi} \sum_{m=1}^{l} \frac{(-1)^{m}(l+m-1) !}{(2 m) !(l-m) !}\left(\frac{4 \pi}{x}\right)^{2 m} B_{2 m},(9)
\end{aligned}
$$

where $B_{2 m}$ are Bernoulli coefficients.

\subsection{The Main Equation}

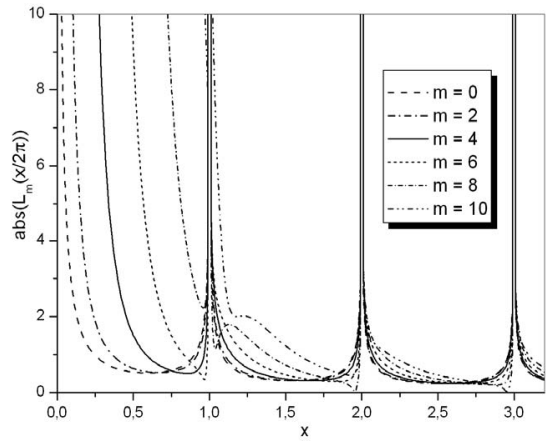

Fig. 2. The lattice sums behavior.

After fulfilling of substitution $b \rightarrow b_{m} / J_{m}(k a)$ and excluding coefficients $\left\{c_{m}\right\}$, we obtain

$$
[I+A(\kappa ; \xi, \alpha)] X=b, \quad A=\left\{A_{m n}\right\}_{m . n=-\infty}^{+\infty} \quad b=\left\{b_{m}\right\}_{m=-\infty}^{+\infty}
$$

where $\kappa=k a, \quad \xi=p / a$, and

$$
\begin{aligned}
& A_{m n}=K_{m n}(\kappa) L_{n-m}(\kappa \xi), K_{m n}(\kappa)=\frac{V_{m}^{E, H}(\kappa) J_{n}(\kappa)}{F_{m}^{E, H}(\kappa) J_{m}(\kappa)} \quad b_{m}=(-1)^{m} \frac{V_{m}(\kappa)}{F_{n}(\kappa)} \\
& V_{m}^{E}=\alpha J_{m}(k a) J_{m}^{\prime}(\alpha k a)-J_{m}^{\prime}(k a) J_{m}(\alpha k a) \quad V_{m}^{H}=\frac{1}{\alpha} J_{m}(k a) J_{m}^{\prime}(\alpha k a)-J_{m}^{\prime}(k a) J_{m}(\alpha k a) \\
& F_{m}^{E}=\alpha H_{m}(k a) J_{m}^{\prime}(\alpha k a)-H_{m}^{\prime}(k a) J_{m}(\alpha k a) \quad F_{m}^{H}=\frac{1}{\alpha} H_{m}(k a) J_{m}^{\prime}(\alpha k a)-H_{m}^{\prime}(k a) J_{m}(\alpha k a)
\end{aligned}
$$

This is the Fredholm second-kind matrix equation due to the rapid enough decrease of the matrix elements $A_{m n}$ if $n, m \rightarrow \infty$. 


\subsection{Floquet Harmonics and Results}

Representation (2) converge rapidly enough, and so we rewrite itd in terms of the Floquet harmonics [8].This is achieved by substituting the Fourier transforms for the Hankel functions

$$
H_{n}\left(k r_{s}\right) e^{i n \varphi_{s}}=\frac{1}{\pi} \int_{-\infty}^{\infty} e^{\left(i k(x-s p) t+i k y \sqrt{1-t^{2}}\right)} \frac{e^{i n \arcsin t}}{\sqrt{1-t^{2}}} d t,
$$

into expression (2) and performing some mathematical manipulations involving the Poisson summation formula. As a result, we obtain the Floquet series in terms of the generalized plane waves,

$$
\begin{aligned}
& U^{s c a t}(x, y)=\sum_{s} f_{s}^{+} e^{i \gamma_{s} k x} e^{i g_{s} k y}, \quad y>d \quad f_{s}^{+}=\frac{2}{k p g_{s}} \sum_{n} b_{n} e^{i n \arcsin \gamma_{s}} \\
& U^{s c a t}(x, y)=\sum_{s} f_{s}^{-} e^{i \gamma_{s} k x} e^{-i g_{s} k y}, \quad y<-d \quad f_{s}^{-}=-\frac{2}{k p g_{s}} \sum_{n} b_{n} e^{i n \arcsin \gamma_{s}}
\end{aligned}
$$

where

$$
\gamma_{s}=\frac{2 s \pi}{k p} \quad g_{b}=\sqrt{1-\gamma_{s}^{2}}
$$

If the normalized frequency $\sigma=\kappa \xi / 2 \pi<1$, the plane wave reflectance is defined as the squared amplitude of the zero Floquet harmonic, $f_{0}^{+}$. The data in Fig. 3 are for the H-polarization and strongly depend on the value of $\sigma$ that is typica for such problems - see, for instance, [13]. Here we see the resonances near to the integer values of the indicated parameter $\sigma$. They are associated with the natural modes of the grid of dielectric wires.

(a)

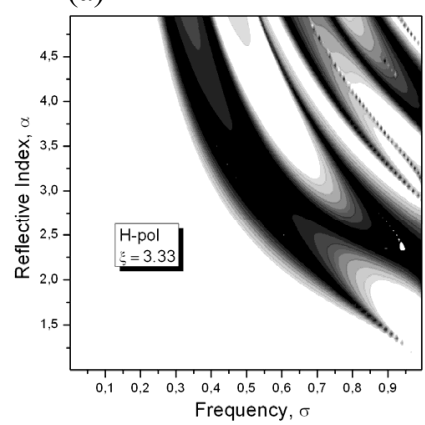

(b)

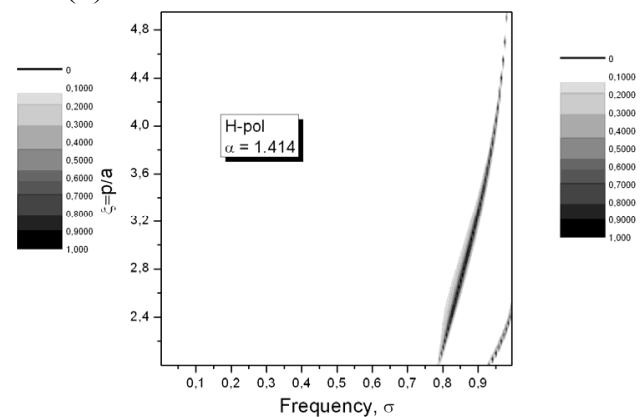

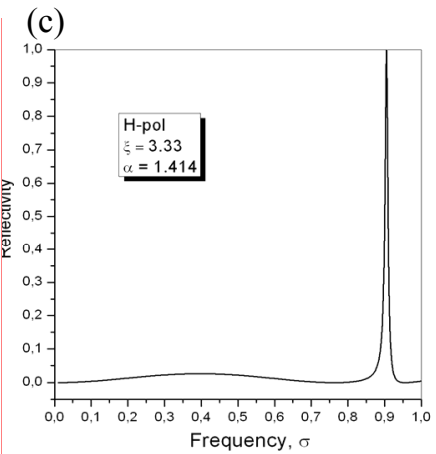

Fig. 3. Reflectance of the periodic chain of dielectric wires under the normal incidence, H-polarization.

\section{EIGENPROBLEM}

Returning to equation (13), omitting the incident field, and introducing "activity" in the cylinders by adding a negative imaginary part to the refractive index, we obtain the characteristic equation for the eigenproblem,

$$
\operatorname{det}[I+A(\kappa ; \xi, v)]=0, \quad A=\left\{A_{m n}\right\}_{m . n=-\infty}^{+\infty},
$$

where the coefficients $\left\{A_{m n}\right\}_{m . n=-\infty}^{+\infty}$ have exactly the same form as (14) and only in (15)-(16), instead of the real refractive index $\alpha$ we substitute the complex number, $v=\alpha-i \gamma$. The determinant equation (20) is very sensitive to convergence of coefficients $\left\{A_{m n}\right\}_{m, n=-\infty}^{+\infty}$. If the unknowns have been chosen properly, the matrix elements decay as

$$
A_{m n} \sim \frac{c \kappa^{2 m} e^{2 m}(n-m)^{n-m-1 / 2}}{\pi^{1 / 2} 2^{n+m+3 / 2} \xi^{n-m} n^{n+1 / 2} m^{m-1 / 2}}, \quad n>>1, m>>1
$$

That is quite enough for satisfying the Fredholm conditions. 
(a)

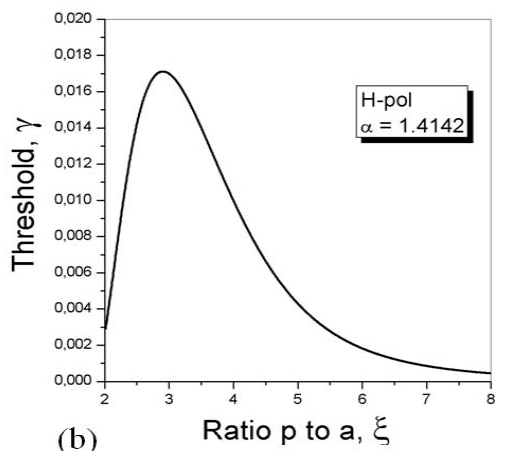

(b)

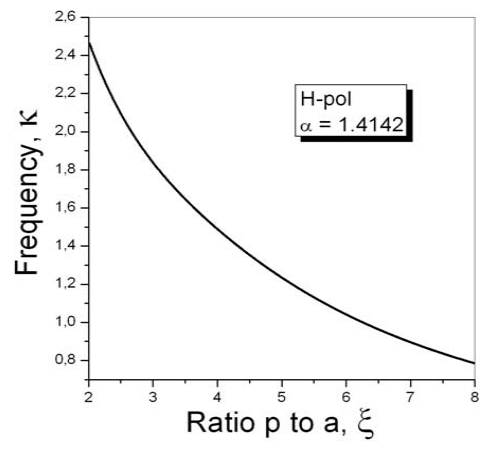

(c)

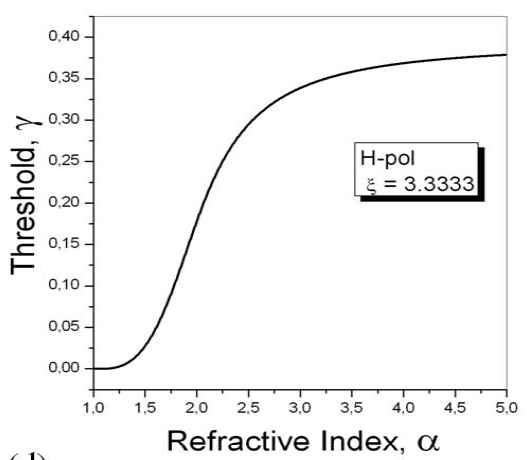

(d)

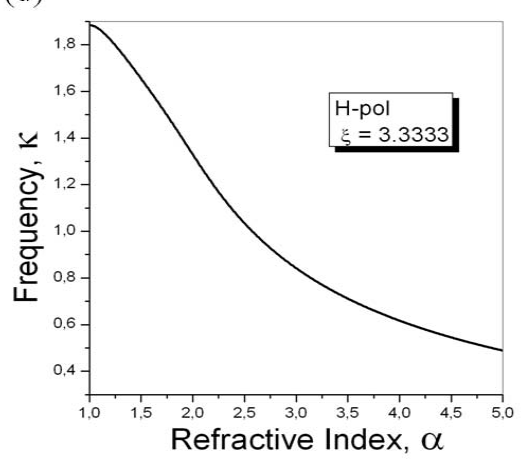

Fig. 4. Eigenparameters of lasing for the infinite periodic chain of active nanowires.

The results in Fig. 4 are obtained by iterations from the first maximum in Fig. 3 (c) taken as the initial guess. Here the most interesting is the plot in Fig. 4 (a), where we observe the drop in the lasing threshold with increasing the value of $\xi$. It means that when the volume of the active region in periodical structure decreases, the threshold value of material gain also gets smaller.

This paradoxical behaviour is linked to the assumption that chain of active nanowires is infinite.

The frequency of lasing tends here to the branch point associated to the grazing propagation of a plane wave given by a Floquet harmonic. This is evident from the lattice sum representations (8) and (9).

\section{ACKNOWLEDGEMENTS}

This work was supported in part by the National Academy of Sciences of Ukraine (NASU) via the project \#07/36-H, the Royal Society, UK via the project IJP-2007/R1-FS, and the TUBITAK and NASU via exchange project.

\section{REFERENCES}

[1] S. F. Yu, "Analysis and Design of Vertical Cavity Surface Emitting Lasers", Wiley, 2003.

[2] A.I. Nosich, E.I. Smotrova, S.V. Boriskina, T.M. Benson, P. Sewell, "Trends in microdisk laser research and linear optical modelling", Optical and Quantum Electronics, vol. 39, no. 15, pp. 1253-1272, 2007.

[3] E.I. Smotrova, A.I. Nosich, "Mathematical analysis of the lasing eigenvalue problem for the WG modes in a 2-D circular microcavity", Optical and Quantum Electronics, vol. 36, no. 1-3, pp. 213-221, 2004.

[4] E.I. Smotrova, A.I. Nosich, T.M. Benson, P. Sewell, "Cold-cavity thresholds of microdisks with uniform and non-uniform gain: quasi-3D modelling with accurate 2D analysis", IEEE J. Selected Topics Quantum Electronics, vol. 11, no. 5, pp. 1135-1142, 2005.

[5] V.O. Byelobrov, A.I. Nosich, "Mathematical analysis of the lasing eigenvalue problem for the optical modes in a layered dielectric microcavity with a quantum well and distributed Bragg reflectors", Optical and Quantum Electronics, vol. 39, no. 10-11, pp. 927-937, 2007.

[6] E.I. Smotrova, et al., Optical coupling of the WG modes of two identical microdisks and its effect on photonic molecule lasing, IEEE J. Selected Topics Quantum Electronics, vol. 12, no. 1, pp. 78-85, 2006.

[7] C.M. Linton, "The Green's function for the two-dimensional Helmholtz equation in periodic domains", J. Engineering Mathematics, vol. 33, pp. 377-402, 1998.

[8] K. Yasumoto, H. Toyama, T. Kushta, "Accurate analysis of 2-D electromagnetic scattering from multilayered periodic arrays of circular cylinders using lattice sums technique", IEEE Trans. Antennas Propagation, vol. 52, no. 10, pp. 2603-2611, 2004. 